\title{
A method for cross-cultural adaptation of a verbal memory assessment
}

\author{
Yen Ying Lim and Khic Houy Prang \\ RMIT University, Bundoora, Australia \\ LuCETTE Cysique \\ University of New South Wales, Sydney, Australia \\ Robert H. Pietrzak \\ Yale University School of Medicine, New Haven, Connecticut \\ and CogState Ltd., Melbourne, Australia \\ Peter J. Snyder \\ Warren Alpert Medical School of Brown University, Providence, Rhode Island \\ AND \\ Paul Maruff \\ CogState Ltd., Melbourne, Australia \\ and University of Melbourne, Parkville, Australia
}

\begin{abstract}
Verbal memory tests—although important to the neuropsychological assessment of memory—are biased to many cultures. In the present article, we highlighted the limitations associated with the direct translation of tests and word matching, as well as the lack of ecological validity and cultural appropriateness when tests developed in one culture are used in another. To overcome these limitations, a verbal memory paradigm was developed that framed the memory assessment with a shopping-list format, but that developed culturally specific stimuli for the different language groups. The aim of the present study was to determine the equivalence of this shopping list memory test in different cultural and language groups. Eighty-three adults from English-, French-, Malay-, and Chinese-speaking cultures participated in four experiments. The results of all the experiments indicated that performance of verbal list learning is equivalent, irrespective of the language used. These results support the use of this methodology for minimizing cross-cultural test bias, and have important implications for testing culturally and linguistically diverse individuals.
\end{abstract}

The assessment of verbal memory is important in neuropsychology, since a range of psychiatric and neurological conditions present with disturbances in the encoding, retrieval, and storage of new verbal information (GrothMarnat, 2003; Lezak, 1995). Verbal memory is most often assessed using tests either of verbal list learning (Lezak, 1995; Lezak, Howieson, \& Loring, 2004) or of the recall of short stories (Lezak, 1995). Although many different verbal memory tests are currently in wide use for clinical and research purposes, they are all similar in that each requires the information that exceeds immediate memory span to be read to the person being tested. This person then attempts to recall as many items from that list, or from the short story, as possible (Lezak, 1995; Mitrushina, Boone, Razani, \& D'elia, 2005). In the case of list-learning tasks, this process is then repeated over short intervals (i.e., learning trials) so that individuals have the opportunity to learn the items over multiple presentations. The outcome measures used most commonly to characterize performance on verbal list-learning tests are therefore the total number of words recalled across all learning trials and the rate at which these words are acquired (Lezak, 1995; Mitrushina et al., 2005). The total number of words recalled is inferred to reflect an individual's ability to learn the verbal information, and the rate of acquisition reflects how fast an individual is able to learn and consolidate new information into memory (Lezak, 1995; Mitrushina et al., 2005).

The analysis of performance on verbal list-learning tests has been extended to include the effects of the serial position of words in the list, as well as the speed with which words are recalled after the list has been read. The serial position effect reflects that words at the middle of the list are not recalled as well as those at the beginning

P. Maruff, pmaruff@cogstate.com 
(i.e., a primacy effect) and end (i.e., a recency effect) (Jung, 1968). Although neuropsychological studies of verbal learning rarely measure the timing of responses, experimental studies show that the rate at which words are recalled decreases with the number of words remembered (i.e., are characterized by a negative logarithmic function) (Murdock \& Okada, 1970; Postman, Egan, \& Davis, 1948; see Figure 1 for an example). Furthermore, the rates of recall also reflect memory strength, with better memory indicated by faster rates of recall (Cowan, 1992; Hulme \& Tordoff, 1989).

Verbal list-learning tests, like all neuropsychological procedures, have been developed primarily within European American cultures (Ardila, 2005; Greenfield, 1997; Rosselli \& Ardila, 2003). Given the importance of verbal list-learning tests to neuropsychological assessment, these tests should ideally be able to be used across different cultures and languages with equivalent validity and reliability. However, neuropsychological measures do not necessarily transfer easily across cultures, especially when such tasks use language-based stimuli or require complex verbal instructions (Ardila, 2005; Greenfield, 1997). Where verbal list-learning tasks have been used to assess memory in different cultures and languages, they have required adaptation to remain meaningful and relevant (see, e.g., Agranovich \& Puente, 2007; Au, Chan, $\&$ Chiu, 2003). Two main approaches have been used to adapt verbal list-learning tests for cross-cultural use. The first approach has been to translate the original English words into the other language. However, direct translation of items may not be possible, since certain languages may lack particular words (Dick, Dick-Muehlke, \& Teng, 2006). For example, ambuyat is a traditional Malay dish

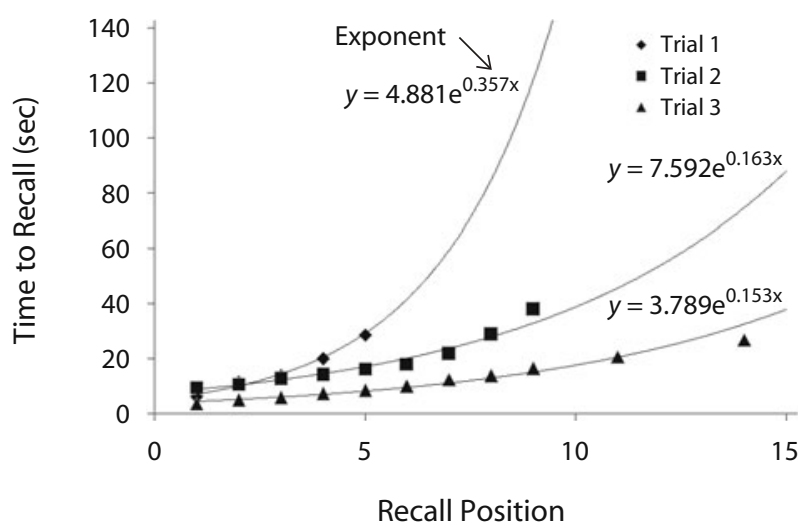

Figure 1. Illustration of the rate of recall as described by Postman et al. (1948), but applied to data from a single subject's performance on the SLT. On the $y$-axis is the time from the end of the reading of the word list. On the $x$-axis is the position of each word recalled. Three data sets reflecting performance on Trials 1, 2, and 3 are shown. An exponential curve has been fitted to the relationship between recall position and time of recall for each of the trials (from Experiment 3, tested in Malay), and the exponent has been computed from this curve. In this example, faster cessation of recall and fewer words recalled (e.g., on Trial 1) are characterized by a larger value for the exponent. Thus, the exponent decreases as recall of the word list improves across trials. made out of sago and has no equivalent in the English language. Furthermore, even if direct translation of the word into a second language is possible, there is a strong possibility that the cultural relevance of the word will be different (Lezak et al., 2004; Matsumoto, 1994). For example, the fruit mangosteen (translated as manggis in Malay) will vary in familiarity between southeast Asian and Australian populations. Although mangosteen is part of the English language and is a concrete noun, it possesses less cultural relevance in the United States or Australia than in southeast Asian countries, in which it is both popular and widely available.

A slightly more sophisticated approach for adapting verbal list-learning tests across cultures has been to establish linguistic similarity for each language by matching words on characteristics that are known to influence recall, such as frequency, familiarity, imaginability, syllable number, or length (Bock \& Klinger, 1986; Glanzer, 1972; Paivio, 1968). Even though these characteristics are not cultural per se, they have been used to guide the development of verbal stimulus sets within cultures and therefore may provide a framework for ensuring the equivalence of word lists across cultures. For example, Agranovich and Puente (2007) selected words for use in their verbal memory test by matching Russian and English words according to their frequency and length. They found that Russian subjects performed better than American subjects on the respective word lists. However, the results did not indicate that Russians were better at remembering items than were American subjects, because even though there was an attempt to match the verbal stimuli between cultures, the differences in test-item familiarity and appropriateness may still have operated differently for the Russian and American groups. This example illustrates an important point: Matching words on individual characteristics, such as frequency and length, does not ensure that other characteristics, such as imaginability or familiarity, will also be equivalent. Words that are high in frequency are commonly assumed to be high in familiarity as well, and yet neither frequency nor familiarity ensures high levels of imaginability (Paivio, 1968). Several studies highlight the importance of ensuring that word imaginability is accounted for, since words high in imaginability are more likely to facilitate recall (Bock \& Klinger, 1986; Paivio, 1968).

Even if one or more word characteristics were sufficient to ensure the cultural equivalence of word lists used in verbal memory tests, tables of word frequency, imaginability, and length that are used to guide the development of lists in English may not be available for other languages. Furthermore, characteristics such as word length are irrelevant in Asian languages such as Mandarin, in which pictorial characters are used instead of an alphabet (e.g., the Roman alphabet). For example, the word bread in English contains one syllable, five letters, and five pen strokes, whereas bread in Mandarin (面包) consists of two syllables, two characters, and 14 brushstrokes. Matching by word characteristics would present with an obvious problem. One extension to the use of word characteristics to guide the selection of words for verbal list-learning tests would be to identify classes of words that have equiva- 
lent valence across cultures as a consequence that they describe species-specific rather than culturally specific characteristics. For example, words to describe family members, body parts, and basic activities of daily living (e.g., eating, obtaining food) are common to humans and therefore should have high imaginability, familiarity, and frequency in all cultures.

An activity important to all humans is that of obtaining food (Rozin, 2007). Although diets differ culturally and regionally, humans seek a wide variety of raw foods and ingredients for cooked foods in order to satisfy their nutritional requirements. Some foods are common across different cultures (e.g., chicken, eggs), whereas other types of foods are culturally and regionally specific (e.g., hummus, ambuyat). Despite these differences, many of the foods used in each cultural group should have high imaginability, high frequency of use, and high familiarity. Therefore, provided that the food words' use can be established to be common within a specific culture, they may provide a useful framework for assembling stimulus sets that are equivalent across cultures.

Aside from the problems of translation and item familiarity, a broader issue with cross-cultural testing is that some cultures have lower levels of experience with test taking generally. When test administrators use tests that have been developed in their own culture and administer it to members of a different culture, the assumptions concerning values, knowledge, and communication are assumed implicitly by the test (Greenfield, 1997). Thus, the results may not reflect the same memory processes in both cultures (Reynolds, 2000; Shiraev \& Levy, 2007). Although people living in developed and Western cultures are generally familiar with taking tests in medical, psychological, and educational contexts, there are subgroups in these cultures who are also less familiar with psychological assessment - for example, people who are elderly, who are from remote regions, who are of low socioeconomic status, who have low educational levels, or who are immigrants (Nell, 2000). Researchers have also argued that for these groups, the reliability of conventional neuropsychological tests - including verbal learning tests - may be undermined because of their limited relevance to day-to-day life, since test items are commonly unrelated (Greenfield, 1997; Youngjohn, Larrabee, \& Crook, 1991).

Part of the solution to developing verbal memory tests that are appropriate for use across cultures/languages is to consider the ecological validity of the test itself. In the context of neuropsychological assessment, ecological validity is the ability of a neuropsychological test to infer and make conclusions about an examinee's behavior in real-world settings (e.g., school, home) from the examinee's performance on the test (Sbordone, 1996). A test that possesses ecological validity is able to approximate real-life situations that are under investigation (Brewer, 2000). Shopping-list contexts have been used previously to increase the ecological validity of verbal list-learning tests - for example, the California Verbal List Test (CVLT) and the Grocery List Selective Reminding Test, as was reported by Youngjohn et al. (1991). Importantly, a test that possesses ecological validity does not necessarily possess cultural appropriateness. For example, although the CVLT uses a shopping-list format to increase the ecological validity of the procedure, the to-be-remembered items on the CVLT remain relevant only to developed or Western cultures (e.g., shorts, glasses, soap, plums). Hence, direct translation of these words so that the CVLT can be used in less-developed cultures will result in a test that uses stimuli that have low familiarity and imaginability.

The arguments that support the ecological validity of the shopping-list format and the potential for common food items to provide a framework for establishing culturally equivalent verbal stimulus sets converge to suggest that the shopping-list format could be an effective paradigm tool for cross-cultural assessment of verbal memory. A verbal memory test that required 12 words to be learned over three trials was recently developed (Pietrzak et al., in press). The stimuli used on this test consisted of food items that were common in the English-speaking United States of America, and the test was presented using the instruction, "Here is a list of items I want you to get from the store, can you remember these and repeat them back to me?" The performance of both healthy adults and adults with chronic schizophrenia on the test was correlated highly with performance on another 12-word, three-trial verbal memory test (The Hopkins Verbal Learning Test [HVLT]). Also, the magnitude of impairment in verbal memory detected in the schizophrenia group (as compared with that in the controls) was equivalent between the shopping-list test (SLT) and the HVLT (e.g., approximately $1 S D$ ). These data suggest that the shopping-list format and the shopping-list items can provide a valid assessment of verbal memory. Thus, the applicability and usability of the test in cultures other than North American can now be determined.

Many studies that have compared performance on verbal list-learning tests between cultures have used only summary measures such as total recall to measure equivalence. Given that multiple measures of memory functions (i.e., acquisition rate, primacy, recency) can be derived even from simple list-learning tests, a thorough examination of cultural equivalence should compare these to determine whether the memory processes that underlie performance on verbal list-learning tests are also equivalent between any cultures. Therefore, the overarching aim of the present series of studies was to determine the extent to which the shopping-list memory test could be extended to cultures other than North American. This was done by developing a process to identify verbal stimuli that are culturally appropriate for use in different geographical regions and then using these stimuli and the shopping-list format to compare verbal memory between different cultural groups. Performance on the verbal memory test was analyzed in terms of the component memory processes so that it was possible to determine which aspects of verbal memory were equivalent between cultural/language groups.

The first experiment compared performance on two SLTs: one that contained items commonly available in the USA but not in Australia, and another that contained items that were common in Australia but not in the USA. The test used groups of people from the USA and Australia. Thus, 
the first experiment compared performance on different versions of the SLT in two different developed countries in which psychological assessment is common and in which the same language is spoken (with minimal variations more so for colloquial than for formal language), but in which there are cultural differences in the foods consumed. The second experiment compared performance between groups of people from France and Australiatwo different developed countries in which psychological assessment is common, but in which the languages are different and there are cultural differences in the foods consumed. The second experiment also tested the assumption that performance on an English-language shopping list that was translated - in this case, into French-would be worse than performance on a shopping list that consisted of items common in France. The third experiment compared performance between groups of people who spoke Mandarin Chinese (hereafter termed "Mandarin") and Malay, and who lived in Brunei-a country in which psychological assessment is not common. Because both language groups live in the same region, however, there are consistencies across cultures in the food consumed. Because the first three studies tested the hypothesis of interest by comparing performance between culture/language samples, the fourth study sought to ensure that differences in demographic or psychological characteristics of the different samples did not affect patterns of differences. This was achieved by comparing different language versions within individuals who were bilingual in Mandarin and Malay and who lived in the same geographical location. The overarching hypothesis was that performance on the shopping lists using culturally and regionally validated items as stimuli would be equivalent between different language and cultural groups.

\section{EXPERIMENT 1}

The aim of the Experiment 1 was to investigate the effect of region on the performance on the shopping-list task by comparing performance on word lists developed for both America and Australia.

\section{Method}

Subjects. A group of 25 adults ( 13 female) between the ages of 20 and 27 years (mean age $=25$ ) was recruited from undergraduate college students at a North American university. All of these subjects were born in North America and had lived there for their entire lives. All of the subjects rated English as their primary language. A group of 25 adults ( 13 females) between the ages of 19 and 27 years (mean age $=25.2$ ) were recruited from undergraduate college students at an Australian university. All of these subjects were born in Australia and had lived there for their entire lives. All rated English as their primary language. No subject was paid.

Word lists. The procedure described in the Appendix was applied in Australia and North America. From this procedure, 12 words were identified by the U.S. sample as easy to obtain and by the Australian sample as difficult to obtain (the U.S. list). Another 12 words were identified as easy to obtain by the Australian sample and as difficult to obtain by the U.S. sample (the Australian list). These word lists are provided in the Appendix. The two word lists were added to the software to form two versions of the SLT for each cultural group - a list of difficult-to-obtain words, and a list of easy-to-obtain words.
SLT. The SLT is a 12-word three-trial verbal list-learning test that is similar in design to the HVLT. For the SLT, the presentation of stimuli and the recording of responses are controlled by a laptop computer and touchscreen. For each 12-word list that is used, the software selects at random the order in which words are presented to subjects. On this test, the subject is seated opposite the experimenter, and the experimenter holds the computer so that the subject cannot see the display. The software prompts the experimenter to say, "In this task, I am going to read you a shopping list. I would like you to remember as many items from this list as possible. Are you ready to start?" When the subject acknowledges that he or she is ready to begin, the experimenter presses the start button, and each of the words in the shopping list are presented one at a time on the display at a rate of 1 word every $2 \mathrm{sec}$. As soon as the new word appears on the screen, the experimenter reads it aloud to the subject. When the 12 items have been read, the software prompts the experimenter to say "Tell me as many of the items on the shopping list as you can remember." At the same time, a list of all the words that have been read to the subject is presented on the display so that only the experimenter can see it. Beside each word is a blank checkbox. The subject recalls as many of the items as he or she can. With each word recalled, the experimenter marks the item on the display using a stylus to touch the relevant checkbox. The number of intrusions made is counted, as is the number of times each word is said. When the subject indicates that he or she can recall no more words, the trial is stopped. The experimenter then says, "I am going to read the same shopping list. Try and remember as many items as you can. Are you ready to start? This entire process is repeated one more time. The shopping-list items on each version of the SLT are presented in the same order on each trial.

Data analysis. For each trial, the number of words recalled correctly was computed. For correctly recalled words, the order in which they were recalled, as well as the time elapsed from the beginning of the recall trial, was recorded. These data were then recombined to provide the main outcome measures, which are the total words recalled across the three trials and the total words recalled on each trial. A primacy score was computed for each subject by summing the number of words from the first three positions on the list recalled on each of the trials (i.e., the maximum score $=9$ ). A recency score was also computed by summing the number of words from the last three positions on the list recalled on each of the trials (i.e., the maximum score $=9$ ). For each subject and for each trial, a rate of recall slope was also generated (see Figure 1 for an example). This was done by plotting the time elapsed from the beginning of the recall trial when each word was recalled (i.e., when it had been checked by the experimenter). For each subject and for each trial, the relationship between the time from the beginning of the trial and the word position was plotted, and an exponential curve fitted. From each curve, the exponent was computed and then used as a measure of the rate of recall in the data analysis. For this measure, higher values reflect a faster decay of the memory trace and therefore worse performance (see Figure 1). The group means for each of these measures under each experimental condition are summarized in Table 1 .

\section{Results and Discussion}

The group means for the data obtained in Experiment 1 are summarized in Table 1. The total words recalled on each version of the SLT for each subject in the USA and Australian groups was submitted to a 2 (culture: USA, Australian) $\times 2$ (list: USA, Australian) ANOVA, with list treated as a repeated factor. The ANOVA indicated a significant interaction between culture and list [Wilks's $\Lambda=0.16, F(1,48)=124.50, p<.001]$. Post hoc tests showed that performance on the USA list was superior in the USA group as compared with that in the Australian group $[t(48)=9.40, p<.001, d=1.82]$, and performance on the Australian list was superior in the Australian 
Table 1

Summary of Results for Experiment 1

\begin{tabular}{|c|c|c|c|c|c|c|c|c|c|c|c|c|}
\hline \multirow{3}{*}{$\begin{array}{l}\text { Performance } \\
\text { Measure }\end{array}$} & \multicolumn{4}{|c|}{ USA List } & \multicolumn{4}{|c|}{ Australia List } & \multicolumn{4}{|c|}{ Data Reorganized } \\
\hline & \multicolumn{2}{|c|}{$\begin{array}{c}\text { USA } \\
(n=25)\end{array}$} & \multicolumn{2}{|c|}{$\begin{array}{l}\text { Australia } \\
(n=25)\end{array}$} & \multicolumn{2}{|c|}{$\begin{array}{c}\text { USA } \\
(n=25)\end{array}$} & \multicolumn{2}{|c|}{$\begin{array}{l}\text { Australia } \\
(n=25)\end{array}$} & \multicolumn{2}{|c|}{$\begin{array}{l}\text { Familiar } \\
(n=50)\end{array}$} & \multicolumn{2}{|c|}{$\begin{array}{l}\text { Unfamiliar } \\
(n=50)\end{array}$} \\
\hline & $M$ & $\overline{S D}$ & $M$ & $S D$ & $M$ & $\overline{S D}$ & $M$ & $\overline{S D}$ & $M$ & $S D$ & $M$ & $S D$ \\
\hline Total Recall & 25.6 & 1.9 & 22.5 & 1.5 & 22.2 & 1.9 & 25.8 & 1.9 & 25.4 & 1.60 & 22.4 & 1.30 \\
\hline Trial 1 & 6.5 & 1.2 & 5.9 & 0.9 & 6.1 & 1.9 & 6.6 & 1.3 & 6.2 & 0.76 & 5.5 & 0.89 \\
\hline Trial 2 & 8.1 & 0.9 & 7.1 & 1.1 & 6.9 & 1.2 & 8.3 & 0. & 8.3 & 0.8 & 6.9 & 0.67 \\
\hline Trial 3 & 11.0 & 1.3 & 9.6 & 0.8 & 10.1 & 0.9 & 11.3 & 1.1 & 10.9 & 0.89 & 9.9 & 0.75 \\
\hline Primacy & 7.4 & 1.9 & 6.5 & 1.6 & 6.9 & 1.6 & 7.7 & 1.5 & 7.9 & 1.6 & 6.8 & 1.5 \\
\hline Recency & 7.6 & 1.4 & 7.3 & 1.9 & 7.4 & 1.2 & 7.3 & 1.1 & 7.4 & 1.9 & 7.6 & 2.1 \\
\hline Rate of Recall & & & & & & & & & 0.26 & 0.08 & 0.29 & 0.07 \\
\hline Rate of Recall Trial 2 & & & & & & & & & 0.21 & 0.07 & 0.24 & 0.10 \\
\hline Rate of Recall Trial 3 & & & & & & & & & 0.21 & 0.09 & 0.23 & 0.09 \\
\hline
\end{tabular}

group as compared with that in the USA group $[t(48)=$ $6.0, p<.001, d=1.9]$. This interaction remained significant when the variability related to trial was added to the model [i.e., a culture $\times$ familiarity interaction, Wilks's $\Lambda=0.28, F(1,48)=1.24, p<.001]$. And, although the effect of trial in this model was significant [Wilks's $\Lambda=$ $0.20, F(2,47)=1.16, p<.001]$, no other two- or threeway interaction was significant.

To decompose the culture $\times$ familiarity interaction, the data were reorganized into performance for both familiar (USA list in USA sample, Australian list in Australian sample) and unfamiliar (Australian list in USA sample, USA list in Australian sample) lists, and the three-way ANOVA was conducted again. This analysis indicated a main effect for familiarity [Wilks's $\Lambda=0.27, F(1,48)=11.24, p<$ $.001, d=2.1$ ] and for trial [Wilks's $\Lambda=0.20, F(2,47)=$ $11.12, p<.001]$. Averaged over word-item familiarity, there were significant improvements in performance from Trial 1 to Trial $2[t(49)=8.7, p<.001, d=0.6]$, and from Trial 2 to Trial $3[t(49)=11.2, p<.001, d=0.9]$. No other main effects or interactions were significant.

An analysis of the primacy and recency data showed that the mean primacy effect was significantly less for the unfamiliar words list than for the familiar words list $[t(49)=6.2, p<.01, d=0.7]$; however, there was no difference between familiar and unfamiliar word lists for recency $[t(49)=0.6, p=.13, d=0.1]$. Data for the rate of recall are shown in Table 1 . A trial $\times$ familiarity effect ANOVA on the rate of recall indicated a significant effect for trial [Wilks's $\Lambda=0.21, F(2,47)=1.39, p<.05$ ], and also for familiarity [Wilks's $\Lambda=0.16, F(2,47)=1.19$, $p<.05]$, but no interaction. Post hoc $t$ tests indicated that, averaged across familiarity, there was a reduction in the rate of recall from Trial 1 to Trial $2[t(49)=3.20, p>$ $.001, d=0.63]$, but no difference in the rate of recall between Trial 2 and Trial $3(d<0.1)$.

The results indicate that low-familiarity word lists were associated with poor performance of approximately the same magnitude in each cultural sample. In both cases, the magnitude of impairment was approximately 0.8 of a standard deviation unit. Despite the poorer performance, the rate at which words were acquired across trials was equivalent for both low- and high-familiarity words. The lower performance for the low-familiarity list arose because the recall of words from the beginning of the list (i.e., primacy) was worse for low-familiarity words. In addition, the rate at which words were recalled was systematically slower for low-familiarity words. Taken together, these data show that even with high imaginability, low-frequency shopping words are associated with poorer memory performance than are highly familiar shopping items. Importantly, performance under the high-familiarity condition was equivalent between the two cultural groups, despite the words lists' being different. These data suggest that, provided shopping-list items are well known, all aspects of memory performance on different English language versions of the SLT are equivalent.

\section{EXPERIMENT 2}

The aim of Experiment 2 was to determine the extent to which equivalence of performance on the SLT could be obtained when the task was administered in two different languages-French and Australian English-using sets of verbal stimuli that were different from one another, but that were highly familiar to the relevant language/cultural group. A second aim was to test the assertion that simple translation of word lists would result in poorer performance. Therefore, a third condition was included in which a French language word list was given that consisted of the words on the Australian list translated directly into French. The hypothesis was that performance on the validated lists would be equivalent between these groups and better than performance on the translated list.

\section{Method}

Subjects. Twenty-five adults ( 13 female) between the ages of 21 and 26 years (mean age $=25$ ) were recruited from undergraduate college students at a French metropolitan university. All of these subjects were born in France and had lived there for their entire lives. All rated French as their primary language. Twenty-five adults (12 females) between the ages of 21 and 28 years (mean age $=25.2$ ) were recruited from undergraduate college students at an Australian university. All of these subjects were born in Australia and had lived there for their entire lives. All rated English as their primary language. No subject had participated previously in an experimental study or an SLT validation sample. No subject was paid.

Procedure. The procedure described in the Appendix was used to identify 12 high-familiarity French shopping-list items. These words were added to the SLT software, and the standard instructions 
translated into French. The Australian list described in Experiment 1 was used in Experiment 2. Both versions of the SLT were administered and scored using the standard instructions. The Australian list was also translated directly into French (see the Appendix). A native French speaker administered the French word lists to the French sample, and a native Australian English speaker administered the word lists to the Australian sample. The two French lists were given on different days, and the order was counterbalanced. The performance outcome measures were as described in Experiment 1.

\section{Results and Discussion}

The data from Experiment 2 were analyzed in two stages. First, performance was compared between the Australian and French speakers using the validated Australian and French words lists. Second, performance was compared between the validated and directly translated French lists in the French speakers. The group means for the data from the validated lists used in Experiment 2 are summarized in Table 2. An independent-groups $t$ test indicated no difference between groups for the mean total words recalled $[t(48)=1.5, p=.14, d=0.12]$. The number of words recalled on each trial for each group was submitted to a 2 (culture, French, Australian) $\times 3$ (trial) ANOVA, with trial as a repeated factor. This analysis indicated a significant effect for trial [Wilks's $\Lambda=0.38$, $F(2,47)=5.95, p<.001]$, but no significant effect of culture and no interaction. Averaged over cultures, there were significant improvements in performance from Trial 1 to Trial $2[t(49)=13.2, p<.001, d=0.6]$, and from Trial 2 to Trial $3[t(49)=15.9, p<.001, d=0.9]$. There were no differences between groups for the primacy $[t(49)=$ $0.18, p=.13, d=0.1]$ or recency $[t(49)=0.18, p=.21$, $d=0.1]$ scores.

The culture $\times$ trial ANOVAs used to analyze the rate of recall indicated that there was a significant effect of trial [Wilks's $\Lambda=0.27, F(2,47)=1.18, p<.05$ ], but no significant effect of culture and no culture $\times$ trial interaction. Post hoc $t$ tests indicated that, averaged over cultures, there was a significant decrease from Trial 1 to Trial 2 $[t(49)=4.50, p<.001, d=1.2]$, but no difference between Trial 2 and Trial $3[t(49)=0.23, p=.11, d=0.1]$.
These data suggest that all aspects of performance on the SLT were equivalent between the French and Australian samples, despite the use of different word lists in different groups of people.

The group means for data from the directly translated French list used in Experiment 2 are summarized in Table 2. A within-groups $t$ test indicated that the mean of total words recalled was significantly less for the directly translated list than for the validated list $[t(24)=8.5, p<$ $.001, d=1.39]$. The number of words recalled on each trial for each group was submitted to a 2 (translation method: translated, validated) $\times 3$ (trial) ANOVA, with trial as a repeated factor. This analysis indicated a significant effect for translation method [Wilks's $\Lambda=0.18, F(2,47)=6.72$, $p<.001$ ] and trial [Wilks's $\Lambda=0.39, F(2,47)=5.43$, $p<.001]$, but no interaction between translation method and trial. Averaged over translation methods, there were significant improvements in performance from Trial 1 to Trial $2[t(49)=11.6, p<.001, d=0.7]$, and from Trial 2 to Trial $3[t(49)=13.8, p<.001, d=0.8]$. Differences between the translation methods were also significant for the measures of primacy $[t(24)=7.1, p<.001, d=1.05]$ and recency $[t(24)=9.8, p<.001, d=1.59]$ scores.

The translation method $\times$ trial ANOVAs used to analyze the rate of recall also showed a significant effect of translation method [Wilks's $\Lambda=0.27, F(2,47)=3.24$, $p<.01$ ] and trial [Wilks's $\Lambda=0.27, F(2,47)=2.23, p<$ $.01]$, but no interaction between the two factors. These data suggest that when attempting to remember a list of words that have been translated directly from English into the native language, the number of words learned, primacy, recency, and the rate of recall are all worse than they are when individuals attempt to learn a list of words that contains items that are highly familiar to them.

\section{EXPERIMENT 3}

In Experiment 2, we showed that performance on the SLT was equivalent for the French- and Australianspeaking subjects. We also showed that direct translation

Table 2

Summary of Results for Experiments 2, 3, and 4

Experiment 2

\begin{tabular}{|c|c|c|c|c|c|c|c|c|c|c|c|c|c|c|}
\hline \multirow{5}{*}{$\begin{array}{c}\text { Performance } \\
\text { Measure }\end{array}$} & \multicolumn{6}{|c|}{ Experiment 2} & & & & & & & & \\
\hline & \multirow{3}{*}{\multicolumn{2}{|c|}{$\begin{array}{c}\text { Australian } \\
\text { List/ } \\
\text { Australian- } \\
\text { English } \\
\text { Speakers } \\
(n=25) \\
\end{array}$}} & \multirow{3}{*}{\multicolumn{2}{|c|}{$\begin{array}{c}\text { French List/ } \\
\text { French } \\
\text { Speakers } \\
(n=25) \\
\end{array}$}} & \multirow{3}{*}{\multicolumn{2}{|c|}{$\begin{array}{c}\text { Translated } \\
\text { Australian } \\
\text { List/French } \\
\text { Speakers } \\
(n=25) \\
\end{array}$}} & \multirow{2}{*}{\multicolumn{4}{|c|}{ Experiment 3}} & \multicolumn{4}{|c|}{ Experiment 4} \\
\hline & & & & & & & & & & & \multirow{2}{*}{\multicolumn{2}{|c|}{$\begin{array}{l}\text { Bilingual } \\
\text { Mandarin } \\
(n=25)\end{array}$}} & \multirow{2}{*}{\multicolumn{2}{|c|}{$\begin{array}{c}\text { Bilingual } \\
\text { Malay } \\
(n=25)\end{array}$}} \\
\hline & & & & & & & \multicolumn{2}{|c|}{$\begin{array}{c}\text { Mandarin } \\
(n=11) \\
\end{array}$} & \multicolumn{2}{|c|}{$\begin{array}{c}\text { Malay } \\
(n=11) \\
\end{array}$} & & & & \\
\hline & $M$ & $S D$ & $M$ & $S D$ & $M$ & $S D$ & $M$ & $S D$ & $M$ & $S D$ & $M$ & $S D$ & $M$ & $S D$ \\
\hline Total Recall & 25.2 & 2.3 & 25.7 & 2.2 & 22.5 & 2.4 & 24.7 & 2.2 & 25.3 & 3.1 & 26.0 & 3.0 & 27.0 & 4.0 \\
\hline Trial 1 & 6.1 & 1.3 & 6.4 & 1.2 & 5.1 & 1.3 & 5.5 & 1.2 & 5.6 & 1.5 & 6.2 & 1.2 & 6.7 & 1.7 \\
\hline Trial 2 & 8.2 & 1.1 & 8.1 & 1.1 & 6.9 & 1.0 & 8.3 & 0.9 & 8.9 & 1.0 & 9.3 & 1.4 & 9.3 & 1.4 \\
\hline Trial 3 & 11.0 & 0.9 & 11.2 & 1.1 & 9.8 & 0.9 & 11.0 & 0.6 & 10.7 & 1.1 & 10.5 & 1.5 & 11.0 & 1.3 \\
\hline Primacy & 7.2 & 1.9 & 7.5 & 2.8 & 5.2 & 1.6 & 6.8 & 1.6 & 7.6 & 0.9 & 7.4 & 1.2 & 7.6 & 1.3 \\
\hline Recency & 7.7 & 2.6 & 7.8 & 2.1 & 5.1 & 1.3 & 7.6 & 2.0 & 7.3 & 0.8 & 7.7 & 1.2 & 7.4 & 1.2 \\
\hline Rate of Recall Trial 1 & 0.30 & 0.12 & 0.31 & 0.13 & 0.39 & 0.19 & 0.32 & 0.20 & 0.29 & 0.11 & 0.28 & 0.11 & 0.25 & 0.08 \\
\hline Rate of Recall Trial 2 & 0.27 & 0.08 & 0.26 & 0.12 & 0.37 & 0.16 & 0.20 & 0.09 & 0.21 & 0.09 & 0.22 & 0.09 & 0.20 & 0.06 \\
\hline Rate of Recall Trial 3 & 0.27 & 0.10 & 0.27 & 0.09 & 0.36 & 0.2 & 0.19 & 0.04 & 0.18 & 0.03 & 0.22 & 0.07 & 0.20 & 0.04 \\
\hline
\end{tabular}


from English to French resulted in a word list that gave rise to substantial (greater than $1 S D$ ) impairment in performance, suggesting that direct translation can result in performance that underestimates true memory ability and that it therefore should not be used. The aim of Experiment 3 was to compare performance on non-Latin-based language versions of the SLT (Mandarin and Malay) in a geographical region in which there is no current neuropsychological framework for assessment (Brunei). The hypothesis was that performance on the SLT would be similar for both Mandarin-speaking and Malay-speaking subjects.

\section{Method}

Subjects. Eleven Malay-speaking adults ( 5 female) between the ages of 18 and 35 years (mean age $=24.09$ ) were recruited from Internet forums and social-networking groups. All of the subjects were born in Brunei, were schooled in Brunei, and rated their place of residence as Brunei. All rated Malay as their primary language. Eleven Mandarin-speaking adults ( 7 females) between the ages of 18 and 35 years (mean age $=23.55$ ) were recruited from Internet forums and social-networking groups. All of the subjects were born in Brunei, were schooled in Brunei and rated their place of residence as Brunei. All rated Mandarin as their primary language. All Malay- and Mandarin-speaking subjects were university educated. No subject had participated previously in an experimental study or an SLT validation sample. No subject was paid.

Procedure. The procedure described in the Appendix was used to identify 12 highly familiar Mandarin shopping-list items (see the Appendix) and 12 highly familiar Malay shopping-list items (Appendix). These words were added to the SLT software and the standard instructions translated into Mandarin and Malay, respectively. Both versions of the SLT were administered and scored using the standard instructions. A native Mandarin speaker administered the word list to the Mandarin sample, and a native Malay speaker administered the test to the Malay sample. The performance outcome measures were as those described in Experiment 1.

\section{Results and Discussion}

The group means for the data for Experiment 3 are summarized in Table 2 (Experiment 3 columns). An independent-groups $t$ test indicated no difference between groups for the mean total words recalled $[t(20)=0.48$, $p=.64, d=0.20]$. The number of words recalled on each trial for each group was submitted to a 2 (language: Mandarin, Malay) $\times 3$ (trial) ANOVA, with trial as a repeated factor. This analysis indicated a significant effect for trial [Wilks's $\Lambda=0.051, F(2,19)=175.83, p<.001$ ], but there was no significant effect of language and no interactions. Averaged over languages, there were significant improvements in performance from Trial 1 to Trial $2[t(21)=$ $-13.66, p<.001, d=-2.91]$, and from Trial 2 to Trial 3 $[t(21)=-10.33, p<.001, d=-2.20]$. There were no differences between groups for the primacy $[t(20)=1.47$, $p=.16, d=0.63]$ or recency $[t(20)=-0.56, p=.58$, $d=-0.24]$ scores

The language $\times$ trial ANOVAs used to analyze the rate of recall indicated that there was a significant effect of trial [Wilks's $\Lambda=0.60, F(2,19)=6.40, p<.05$ ], but no significant effect of language and no language $\times$ trial interaction. Post hoc $t$ tests indicated that, averaged over languages, there was a significant decrease from Trial 1 to Trial $2[t(21)=3.66, p=.001, d=0.78]$, but no differ- ence between Trial 2 and Trial $3[t(21)=1.10, p=.28$, $d=0.23$. These data suggest that all aspects of performance on the SLT were equivalent between Mandarinspeaking and Malay-speaking samples, despite the use of different word lists in different groups of people.

\section{EXPERIMENT 4}

In Experiment 3, we showed that performance on the SLT was similar for Mandarin- and Malay-speaking subjects. Although valid for establishing the appropriateness of the verbal stimuli, Experiments 1,2 and 3 did not control interindividual variables, such as motivation and concentration, or true differences in memory abilities between the two groups. The use of different groups of people to compare performance between different versions of the SLT is potentially suboptimal, and an assessment of the equivalence of memory performance on the different shopping lists in bilingual people living in a multicultural society could determine the extent to which any demographic, personal, or cognitive factors influenced performance in different groups assessed using different language versions of the SLT. Our aim in Experiment 4 was thus to compare performance on different language versions of the SLT (Mandarin and Malay) in a group that was fluent in both languages (i.e., bilinguals). The hypothesis was that performance across all measures of memory would be equivalent when subjects were tested in Malay and Mandarin.

\section{Method}

Subjects. Twenty-five bilingual (Mandarin-Malay) adults (18 female) between the ages of 18 and 35 years (mean age $=24.64$ ) were recruited from Internet forums and social-networking groups. All subjects were born in Brunei, were schooled in Brunei, and rated their place of residence as Brunei. All subjects had achieved at least a credit grade for their final year of high school (O levels) in both Malay and Mandarin language subjects. All bilingual subjects were university educated. No subject had participated previously in an experimental study or an SLT validation sample. No subject was paid.

Procedure. The Mandarin and Malay lists used in Experiment 3 were used. Both versions of the SLT were administered and scored using the standard instructions. A native bilingual speaker administered the word list to the bilingual sample. The order in which lists were administered was pseudorandomized between subjects. The performance outcome measures were as those described in Experiment 1.

\section{Results and Discussion}

The group means for the data for Experiment 4 are summarized in Table 2 (Experiment 4 columns). An independent-groups $t$ test indicated no difference between groups for the mean total words recalled $[t(48)=0.91$, $p=.37, d=0.27]$. The number of words recalled on each trial for each group was submitted to a 2 (language: Mandarin, Malay) $\times 3$ (trial) ANOVA, with trial as a repeated factor. This analysis indicated a significant effect for trial [Wilks's $\Lambda=0.92, F(2,19)=2.00, p=.15]$, but no significant effect of language, and no interactions. Averaged over languages, there were significant improvements in performance from Trial 1 to Trial $2[t(49)=-15.27, p<.001$, 
$d=-2.16]$, and from Trial 2 to Trial $3[t(49)=-9.73$, $p<.001, d=-1.38]$. There were no differences between groups for the primacy $[t(48)=0.80, p=.43, d=0.23]$ or recency $[t(48)=-0.82, p=.42, d=-0.23]$ scores.

The language $\times$ trial ANOVAs that was used to analyze the rate of recall indicated that there was a significant effect of trial [Wilks's $\Lambda=0.78, F(2,47)=6.48, p<.05$ ], but no significant effect of language and no language $\times$ trial interaction. Post hoc $t$ tests indicated that averaged over languages, there was a significant decrease from Trial 1 to Trial $2[t(49)=3.50, p=.001, d=0.50]$, but no difference between Trial 2 and Trial $3[t(49)=0.10, p=.93, d=$ $0.01]$. These data suggest that all aspects of performance on the SLT were equivalent in bilingual (Mandarin-Malay) samples, despite the use of different word lists.

\section{GENERAL DISCUSSION}

When considered together, the results from this set of experiments show that it is possible to develop tests of verbal list learning that have equivalence across different cultural and language groups, irrespective of the language used and the level of experience with psychological testing in different cultural groups. This can be achieved if word-list memory tests are presented as a shopping list and the stimuli used in these tests consist of items that are common and therefore familiar in the culture under investigation. In Experiment 1, we showed that even when language and experience with psychological assessment was equivalent across cultures, the use of verbal stimuli that are less common in one culture gives rise to substantially poorer performance on word list-learning tests. Furthermore, in Experiment 1, the relatively large difference in memory performance between groups occurred despite all of the verbal stimuli being concrete nouns that have high imaginability. Thus, differences in performance on the shopping-list test reflect familiarity with the verbal stimuli rather than with any aspect of the language or culture itself. When data were reorganized so that familiar words were compared with unfamiliar words irrespective of culture, the poorer performance for unfamiliar words was shown to arise from a diminished primacy effect. In list-learning paradigms, primacy effects are hypothesized to reflect the extent to which information can be encoded into long-term memory (Baddeley, 1986). The rate at which words were acquired across trials was the same for both familiar and less-familiar words; therefore, the reduced encoding associated with the less-familiar list operated systematically across learning trials. These data indicate that even within the same language, the use of less familiar words on a verbal list-learning test interferes generally with the encoding of information into verbal memory. Importantly, when the words in the list were of equivalent familiarity to the individuals from the different cultural groups, the rates of acquisition, primacy, and recency, and the rates of recall were equivalent despite the words themselves being different.

In Experiment 2, we confirmed the hypothesis that despite the use of different words, performance on the shopping-list test would be equivalent across cultures, provided that the words used as stimuli were familiar to the individuals from the different cultural groups. The total recall score, the rate of acquisition, primacy and recency effects, and the rates of recall were all equivalent in the Australian and French samples when they were assessed with Australian-English and French versions of the SLT, respectively. However, when assessed using an Australian shopping list that was directly translated into French, performance in the French speakers was more than one standard deviation below that observed for the validated French list. This is consistent with the findings of Experiment 1, in which familiarity with the verbal stimuli resulted in improved performance, and thus confirms the assertion that direct translation does not provide the equivalence of word lists between cultures (Van de Vijver $\&$ Tanzer, 2004). Since this assertion has already been established in Experiments 1 and 2, comparisons of familiar and nonfamiliar words in English, Malay, and Mandarin were not conducted for Experiments 3 and 4.

In Australian and French cultures, psychological assessment is common; therefore, in Experiment 3, we tested the hypothesis that performance on the verbal list-learning test that was organized as a shopping list could be equivalent across cultures by assessing performance in groups of people from a country in which psychological testing is not common, and in which European-based languages are not spoken. The results of this experiment showed that when the words were classified as being common to each cultural group and the test was presented as a shopping list, measures of the total recall, rate of acquisition, primacy, recency, and rates of recall were equivalent between Malay and Mandarin-Chinese versions of the list.

In the first three experiments, different groups of people were used to compare the different cultural versions of the SLT. Therefore, it is possible that sampling error could have operated to alter performance and led to the patterns of equivalence in performance. For example, the samples of French- or Mandarin-speaking adults could have consisted of people who were interested in research and were therefore more motivated to perform well, whereas the college students in the USA or Australia who were more used to participating in research studies may have been less motivated to perform as well. As such, even if the test was not culturally appropriate, differences in examinees' levels of interest or motivation may have acted to overcome this bias. One way to ensure that individual differences did not have an impact on the results was to compare performance on different word lists that reflected different cultural and language backgrounds in the same people. In Experiment 4, we compared performances on the Malay and Mandarin versions of the SLT in a group of people who had lived their entire lives in the same region, but who were bilingual. The results of this study also showed that measures of total recall, the rate of acquisition, primacy, recency, and rates of recall were equivalent between different language versions of the shopping list, despite the words' on each test used being completely different from one another.

Together, the results from this set of experiments demonstrate that framing a word list-learning test as an SLT 
minimizes the potentially limiting effects of item familiarity and test-taking experience on the performance on memory tests adapted for other cultures. Regardless of culture and language, a person with no history of test taking would be able to comprehend and relate to the requirements of the test (remembering what food items to get) without changing the construct under investigation (verbal memory), since the attainment of food is a central activity of life. The shopping-list method has been used previously to conduct memory assessment in individuals with low familiarity for neuropsychological assessment (e.g., older people, Delis, Kramer, Kaplan, \& Ober, 1987; Delis et al., 1991; Youngjohn et al., 1991). These studies showed that despite the deviation from the more formal and conventional neuropsychological test methodology usually used for word-list learning (e.g., HVLT, Brandt, 1991; Rey Auditory Verbal Learning Test, Rey, 1964), the shopping-list format was well accepted in older people and was also sensitive to the memory impairment associated with dementia (Delis et al., 1991; Youngjohn et al., 1991). The use of a common everyday format for assessing memory has also been formalized in the Rivermead Behavioural Memory Test (RBMT). Although this battery does not include an SLT it does assess memory for newspaper stories, face-name associations, and memory for the location of common objects and tasks (Wilson, Cockburn, \& Baddeley, 1985). The RBMT has been proven to be sensitive for identifying and characterizing impairment in memory in different disorders using the more ecologically relevant approach (Guaiana, Tyson, \& Mortimer, 2004; Jambaqué et al., 2007; Küçükdeveci, Kutlay, Elhan, \& Tennant, 2008). For the USA and Australian groups, the results of the present studies are therefore consistent with these previous studies in showing that ecologically valid testing formats can be used to make valid assessments of memory. When taken together, the results of the series of experiments indicate that ecologically relevant contexts are also useful for adaption of memory tests across cultures.

The use of shopping items rated by a validation sample as being relatively easy to obtain ensured that the stimuli selected for use were well known to people from the specific language and cultural group. The present studies showed that, instead of using unrelated concrete nouns as verbal stimuli (e.g., HVLT; Brandt, 1991), translating words from English, or matching words on the basis of frequency and length, a word list made up of food items that are easily obtained in local markets, stores, or supermarkets in the region in which testing takes place ensures that items will be of equivalent salience for each culture assessed. This is most probably because when considered from a linguistic perspective, the nouns used in the various lists of common food items were uniformly of high frequency, high imaginability, and high familiarity (Bock \& Klinger, 1986; Youngjohn et al., 1991). Thus, even though the words themselves were not the same, the items had equivalent salience for each culture, and this minimized the limitations that are recognized to occur with direct translation and matching on factors such as word and item familiarity across cultures or language groups (e.g., Agranovich \& Puente, 2007).

The present studies provide a strong basis for the development of tools to examine and compare verbal memory between different cultural and language groups. The SLT can be modified to various cultures or even between regions within the same country when there are substantial language variations, with culturally inappropriate items being readily replaced with culturally appropriate ones using the same process as was described in the present article (Appendix). Thus, the SLT possesses the flexibility that most tests of verbal list learning do not have, while maintaining cultural equivalence and avoiding translation difficulties. These data therefore suggest that with the appropriate selection of stimuli, the SLT can be considered an International SLT.

There are several issues that limit the extent to which these results can be applied. The small sample size of each experiment in the present study may have resulted in a lack of power, and the detection of potential differences may have been overlooked. Furthermore, individuals who participated in all experiments were young, healthy adults who were university educated. However, because common food items are used in the test, it is speculated that individuals with low educational levels will perform similarly to university-educated individuals of equivalent age. Nonetheless, this will be of interest to future investigations, especially in illiterate groups.

Before the SLT can be used with a clinical population, further studies need to be conducted to determine that the SLT is sensitive to impairment; that is, individuals suffering from memory impairment will perform significantly worse than healthy controls. Moreover, normative data and frequency of error type will need to be developed before the SLT is used in a clinical setting. Nevertheless, the findings of the present study provide further support for the use of the SLT paradigm for minimizing cross-cultural test bias, thus having important implications when culturally and linguistically diverse individuals are tested.

In conclusion, the universality of neuropsychological tests should not be assumed. Score differences observed in cross-cultural comparisons may be mediated by various factors, and acceptance of such factors as true will invalidate the test and have consequences for diagnosis and intervention. The present study has demonstrated that the SLT is a valid paradigm for use in verbal memory tests across different cultures, since issues that are associated with cross-cultural testing (such as translation difficulties and cultural inappropriateness) and that are commonly faced by other verbal list-learning tests were avoided. Through the investigation of cognitive functions underlying the attainment of total scores, the present study has established that the processes by which verbal memory is acquired are similar between cultures.

\section{AUTHOR NOTE}

Address correspondence to P. Maruff, CogState Ltd., Level 7, 21 Victoria St., Melbourne, Victoria 3000, Australia (e-mail: pmaruff@) cogstate.com). 


\section{REFERENCES}

Agranovich, A. V., \& Puente, A. E. (2007). Do Russian and American normal adults perform similarly on neuropsychological tests? Preliminary findings on the relationship between culture and test performance. Archives of Clinical Neuropsychology, 22, 273-282.

Ardila, A. (2005). Cultural values underlying psychometric cognitive testing. Neuropsychology Review, 15, 185-195.

Au, A., Chan, A. S., \& CHIU, H. (2003). Verbal learning in Alzheimer's disease. Journal of the International Neuropsychological Society, 9 , 363-375.

BADDELEY, A. D. (1986). Working memory. New York: Oxford University Press.

BocK, M., \& KLINGER, E. (1986). Interaction of emotion and cognition in word recall. Psychological Research, 48, 99-106.

BRANDT, J. (1991). The Hopkins Verbal Learning Test: Development of a new memory test with six equivalent forms. Clinical Neuropsychologist, 5, 125-142.

BrEwER, M. (2000). Research design and issues of validity. In H. Reis \& C. Judd (Eds.), Handbook of research methods in social and personality psychology (pp. 3-16). Cambridge: Cambridge University Press.

CowAN, N. (1992). Verbal memory span and the timing of spoken recall. Journal of Memory \& Language, 31, 668-684.

Delis, D. C., Kramer, J. H., Kaplan, E., \& Ober, B. A. (1987). California Verbal Learning Test: Adult version manual. San Antonio, TX: Psychological Corporation.

Delis, D. C., Massman, P. J., Butters, N., Salmon, D. P., Cermak, L. S., \& Kramer, J. H. (1991). Profiles of demented and amnesic patients on the California Verbal Learning Test: Implications for the assessment of memory disorder. Psychological Assessment, 3, 19-26.

Dick, M. B., Dick-Muehlke, C., \& Teng, E. L. (2006). Assessment of cognitive status in Asians. In G. Yeo \& D. Gallagher-Thompson (Eds.), Ethnicity and the dementias (2nd ed., pp. 55-69). New York: Taylor \& Francis

Glanzer, M. (1972). Storage mechanisms in recall. In K. W. Spence \& J. T. Spence (Eds.), The psychology of learning and motivation (Vol. 5, pp. 129-193). New York: Academic Press.

GREENFIELD, P. M. (1997). You can't take it with you: Why ability assessments don't cross cultures. American Psychologist, 52, 1115-1124.

Groth-Marnat, G. (2003). Handbook of psychological assessment (4th ed.). Hoboken, NJ: Wiley.

Guainna, G., Tyson, P., \& Mortimer, A. M. (2004). The Rivermead Behavioral Memory Test can predict social functioning among schizophrenic patients treated with clozapine. International Journal of Psychiatry in Clinical Practice, 8, 245-249.

Hulme, C., \& TordofF, V. (1989). Working memory development: The effects of speech rate, word length, and acoustic similarity on serial recall. Journal of Experimental Child Psychology, 47, 72-87.

Jambaqué, I., Dellatoles, G., Fohlen, M., Bulteau, C., Watier, L., DORFMÜller, G., ET AL. (2007). Memory functions following surgery for temporal lobe epilepsy in children. Neuropsychologia, 45, 2850-2862.

Jung, J. (1968). Verbal learning. New York: Holt, Rinehart \& Winston.
KüÇüKdeveci, A. A., Kutlay, S., Elhan, A. H., \& Tennant, A. (2008). Construct validity and reliability of the Rivermead Behavioural Memory Test in the Turkish population. Brain Injury, 22, 75-82.

LEZAK, M. D. (1995). Neuropsychological assessment (3rd ed.). New York: Oxford University Press.

LezaK, M. D., Howieson, D. B., \& Loring, D. W. (2004). Neuropsychological assessment (4th ed.). New York: Oxford University Press.

Matsumoto, D. (1994). Introduction. In D. Matsumoto (Ed.), People Psychology from a cultural perspective (pp. 1-16). Pacific Grove, CA: Brooks-Cole.

Mitrushina, M., Boone, K. B., Razani, J., \& D'elia, L. F. (2005). Handbook of normative data for neuropsychological assessment (2nd ed.). New York: Oxford University Press.

MuRdock, J. R., \& OKADA, R. (1970). Interresponse times in single-trial free recall. Journal of Experimental Psychology, 86, 263-267.

Nell, V. (2000). Cross-cultural neuropsychological assessment: Theory and practice. Mahwah, NJ: Erlbaum.

PAIVIO, A. (1968). A factor-analytic study of word attributes and verbal learning. Journal of Verbal Learning \& Verbal Behavior, 7, 41-49.

Pietrzak, R. H., Olver, J., Norman, T., Piskulic, D., Maruff, P., \& SNYDER, P. J. (in press). A comparison of the CogState Schizophrenia Battery and the Measurement and Treatment Research to Improve Cognition in Schizophrenia (MATRICS) battery in assessing cognitive impairment in chronic schizophrenia. Journal of Clinical \& Experimental Neuropsychology.

Postman, L., Egan, J. P., \& Davis, J. (1948). Rate of recall as a measure of learning: The effects of retroactive inhibition. Journal of Experimental Psychology, 38, 535-546.

Rey, A. (1964). L'examen clinique en psychologie. Paris: Press Universitaire de France.

REYNOLDS, C. R. (2000). Methods for detecting and evaluating cultural bias in neuropsychological tests. In E. Fletcher-Janzen, T. L. Strickland, \& C. R. Reynolds (Eds.), Handbook of cross-cultural neuropsychology: Critical issues in neuropsychology (pp. 249-286). New York: Springer.

Rosselli, M., \& Ardila, A. (2003). The impact of culture and education on nonverbal neuropsychological measurements: A critical review. Brain \& Cognition, 52, 326-333.

RozIN, P. (2007). Food and eating. In S. Kitayama \& D. Cohen (Eds.), Handbook of cultural psychology (pp. 391-415). New York: Guilford.

Sbordone, R. J. (1996). Ecological validity: Some critical issues for the neuropsychologist. In R. J. Sbordone \& C. J. Long (Eds.), Ecological validity of neuropsychological testing (pp. 15-42). Boca Raton, FL: CRC Press.

Shiraev, E., \& Levy, D. (2007). Cross-cultural psychology: Critical thinking and contemporary applications. New York: Pearson.

VAN DE ViJver, F., \& TANZER, N. K. (2004). Bias and equivalence in cross-cultural assessment: An overview. Revue Européenne de Psychologie Appliquée, 54, 119-135.

Wilson, B., Cockburn, J., \& Baddeley, A. (1985). The Rivermead Behavioural Memory Test. Reading, U.K.: Thames Valley Test Co.

Younguohn, J. R., Larrabee, G. J., \& Crook, T. H. (1991). First-last names and the grocery list selective reminding test: Two computerized measures of everyday verbal learning. Archives of Clinical Neuropsychology, 6, 287-300. 


\section{APPENDIX}

Methodology for Obtaining Culturally Relevant Stimuli

For a specific list, an individual from the culture of interest (who also spoke English fluently and who understood psychological testing) was engaged. This expert was given the USA-English word list and was instructed to identify shopping-list items that were available commonly in the culture of interest. Once this expert had recommended a list of possible items, these were assembled into a survey. On this survey, each word was presented in a list, and subjects were instructed to respond to each item in accord with the following statement: "Please indicate how easy it is to obtain the following items." Next to each item, a 4-point Likert scale was presented, and the descriptors for each gradation on this scale were very easy, easy, difficult, and very difficult. Subjects could select only one possible rating.

Once constructed, the questionnaire was then distributed to 30 adults who lived in the culture of interest. Subjects were defined as "locals" if they were born in, were schooled in, and had rated their place of residence as the particular country of interest. In addition, they had to rate the main language of the culture of interest as their primary language.

The questionnaire was distributed in printed form and electronically through SurveyMonkey. Items that were rated as very easy were coded 1, easy were coded 2, difficult were coded 3, and very difficult were coded 4. A median score was then calculated for each item. Items with a median score of 4 (very difficult) were excluded from the final list. From the final list of words, 12 exemplars were chosen for each of the shopping lists used in this study. These are shown in Table A1.

Table A1

Word Lists Used in the Four Experiments in the Present Study

\begin{tabular}{|c|c|c|c|c|c|}
\hline $\begin{array}{l}\text { English List/ } \\
\text { USA-English } \\
\text { Speakers }\end{array}$ & $\begin{array}{l}\text { English List/ } \\
\text { Australian- } \\
\text { English } \\
\text { Speakers }\end{array}$ & $\begin{array}{l}\text { French List/ } \\
\text { French } \\
\text { Speakers }\end{array}$ & $\begin{array}{c}\text { French List Translated } \\
\text { From Australian/French } \\
\text { Speakers }\end{array}$ & $\begin{array}{c}\text { Mandarin } \\
\text { List/Brunei } \\
\text { Mandarin } \\
\text { Chinese } \\
\text { Speakers }\end{array}$ & $\begin{array}{l}\text { Malay List/ } \\
\text { Brunei } \\
\text { Malay } \\
\text { Speakers }\end{array}$ \\
\hline Fudge & Pavlova & Café & Pavlova & 咖啡 & Kopi \\
\hline Brownies & Flake & Pommes & Flocon & 苹果 & Epal \\
\hline Candy & Lollies & Steak & Sucettes & 牛肉 & Daging \\
\hline Bagel & Pastie & Saumon & Pastie & 鲅鱼 & Ikan \\
\hline Pretzel & Lasagne & Oignons & Lasagne & 蘑菇 & Jagung \\
\hline Ketchup & Vegemite & Chocolat & Vegemite & 薯片 & Keropok \\
\hline Pepperoni & Dim Sim & Champignons & Faible Sim & 面粉 & Nasi \\
\hline Pickle & Flathead & Vinaigre & À tête plate & 胡椒 & Cili \\
\hline Oatmeal & Weetabix & Crêpes & Weetabix & 沙爹 & Satay \\
\hline Soda & Mandarin & Fromage & Mandarin & 鸡蛋 & Telur \\
\hline Cornbread & Crumpets & Bonbon & Crumpets & 人葠 & Ambuyat \\
\hline Syrup & Potato Cake & Poivre & Gâteau de pomme de terre & 豆奶 & Madu \\
\hline
\end{tabular}

Note-Word lists for each country are not direct translations, but have resulted from the protocol detailed in the Appendix.

(Manuscript received February 1, 2009;

revision accepted for publication May 11, 2009.) 